\title{
Correlation of CT signs with lymphatic metastasis and pathology of neuroblastoma in children
}

\author{
XINXIAN ZHANG, CHENGLONG LI, CHAO XU, XIANGDONG HAO, XIAO YU and QIANCHENG LI \\ Department of Radiology, Xuzhou Children's Hospital, Xuzhou, Jiangsu 221000, P.R. China
}

Received November 27, 2017; Accepted May 15, 2018

DOI: $10.3892 / 01.2018 .8959$

\begin{abstract}
Correlation between computed tomography (CT) signs, lymphatic metastasis and pathological features of neuroblastoma (NB) in children was investigated. A total of 374 child patients diagnosed with NB via CT scan and pathological section in Department of Pediatric of Xuzhou Children's Hospital from March 2011 to January 2017 were collected, and their clinical data were retrospectively analyzed. According to CT signs, NB calcification and invasion to surrounding tissues were evaluated, and the tumor site, tumor size, lymphatic metastasis, pathological types and clinical prognosis were analyzed. In plain CT scan, 160 cases showed clear tumor mass, and 214 cases showed blurred mass; 78 cases of tumors were uniform in density, and 296 cases were not uniform in density. Besides, there were 351 cases of calcification in mass. There were 106 cases of axial rotation of kidney, 53 cases of enlargement of renal calyce and renal pelvis, 66 cases of elevation of liver position, 71 cases of pancreas translocation, 26 cases of gastrointestinal tract translocation, 17 cases of vascular translocation and 12 cases of bladder translocation, besides 23 of the cases showed no significantly abnormal changes. Moreover, 211 cases had retroperitoneal lymphatic metastasis with soft tissue swelling in phrenic angle, abdominal aorta and renal hilum in image, and non-uniform annular enhancement or uniform enhancement in enhanced scanning. NB in right adrenal gland invaded the liver in 53 cases, invaded the kidney in 26 cases, invaded the psoas in 40 cases and blood vessels in 32 cases, and the remaining cases showed no invasion. A total of 68 cases were accompanied by pleural thickening, 34 cases by pleural effusion, 36 cases by tracheal compression, 38 cases by rib compression, and 40 cases by tumor invading into vertebral canal. Bone metastasis occurred in 182 cases; liver metastases occurred in 28 cases, and brain metastases in 35 cases. NB
\end{abstract}

Correspondence to: Dr Qiancheng Li, Department of Radiology, Xuzhou Children's Hospital, 18 Sudi North Road, Xuzhou, Jiangsu 221000, P.R. China

E-mail: xinxian777@yeah.net

Key words: children, neuroblastoma, tomography, lymphatic metastasis, pathological type calcification was significantly correlated with pathological type, tumor site and lymphatic metastasis $(\mathrm{p}<0.05)$, but not correlated with tumor size $(\mathrm{p}>0.05)$; NB invasion to surrounding tissues was associated with pathological type, tumor site and clinical prognosis $(\mathrm{p}<0.05)$, but was not correlated with the tumor size $(\mathrm{p}>0.05)$. We concluded that patients with distal mediastinal mass identified by CT examination, accompanied by calcification, and invasion to surrounding tissues may suffer from NB. Tumor growth is closely correlated with tumor differentiation degree.

\section{Introduction}

Neuroblastoma (NB) is a common malignancy in children aged below 8 years, and its incidence is about $1 / 80,000$ worldwide, which is only lower than that of nephroblastoma (1). NB mainly occurs in the retroperitoneal, postmediastinal, inferior cervical, posterior pelvic wall and adrenal medullary sympathetic nervous system, among which retroperitoneal $\mathrm{NB}$ as the most common type accounts for about $70 \%$ of all cases (2). Treatment outcomes of retroperitoneal NB and its prognosis are usually poor compared with other types of NB (3). NB usually shows no obvious characteristics in early stage, and is indistinguishable from flu and fever, and is easily ignored, therefore, most patients are diagnosed at advanced stages (4). Shinagawa et al (5) reported that the mortality rate of stage-IV NB patients within 4 years is up to $65 \%$. Surgery combined with drug therapy has been widely applied in the clinical treatment of NB, but radical treatments are lacking, so the diagnosis and treatment of NB now is a hot research topic worldwide (6). Diagnostic values of computed tomography $(\mathrm{CT})$ for NB have been well studied $(7,8)$, but the correlation between CT signs and pathological features of child patients with NB has not been reported. Therefore, in this study, CT signs of child patients with NB were retrospectively analyzed, and their correlation with pathological features of CT was explored with an expectation of providing reference and guidance for the clinical diagnosis and treatment of NB in the future.

\section{Materials and methods}

General data. A total of 374 child patients diagnosed with NB via CT scan and pathological examination in Department of Pediatric of Xuzhou Children's Hospital from 
Table I. CT parameter setting.

\begin{tabular}{lccccc}
\hline Tube current & Tube voltage & Layer thickness & Interlayer space & Spiral space & Reconstruction interval \\
\hline $130-190 \mathrm{~mA}$ & $130-150 \mathrm{kV}$ & $3 \mathrm{~mm}$ & $1.5 \mathrm{~mm}$ & 1.1 & $1.5 \mathrm{~mm}$ \\
\hline
\end{tabular}

CT, computed tomography.

March 2011 to January 2017 were collected, and their clinical data were retrospectively analyzed. Those patients included 216 males and 158 females, and the age ranged from 4 months to 8 years with a median age of 3.6 years.

Inclusion and exclusion criteria. Inclusion criteria: Patients received CT scan during the treatment in Xuzhou Children's Hospital (Xuzhou, Jiangsu) and with complete imaging data; patients pathologically diagnosed with NB in the hospital; patients with complete medical records; patients strictly followed the instructions of the doctors during treatment. Exclusion criteria: Patients complicated with other cardiovascular and cerebrovascular diseases; patients complicated with digestive tract diseases; patients used antibiotics before admission; anemia patients. The family members of the patients signed informed consent. This study was approved by the Ethics Committee of Xuzhou Children's Hospital (Xuzhou, Jiangsu).

Methods. All child patients underwent continuous volume cranial scanning using Philips Brilliance 16-slice spiral CT scanner ((Philips, Amsterdam, Holland). CT parameter settings are shown in Table I. Plain and enhancement scans were performed from diaphragmatic dome to the pubic symphysis of child patients with iohexol as the contrast agent. Original image data were sent to the ADW4.5 Workstation for multi-planar reconstruction (MPR), volume rendering (VR) and curved planar reconstruction (CPR). Tumor tissue sections of patients were fixed in $10 \%$ formaldehyde, followed by paraffin embedding, and hematoxylin and eosin staining. Immunohistochemical staining was also performed using neuron-specific enolase (NES), S-100 protein (S-100), synaptophysin (Syn) and chromogranin A (CgA). According to CT signs, NB calcification and invasion to surrounding tissues were evaluated by microscope (Olympus, Tokyo, Japan), and the tumor site, tumor size, lymphatic metastasis, pathological type and clinical prognosis were analyzed.

Statistical analysis. Statistical Product and Service Solutions (IBM Corp., Armonk, NY, USA) 22.0 software was used for statistical analysis of data. Enumeration data were presented as rate, and processed using Chi-square test. Correlation analysis was performed using logistic regression analysis. $\mathrm{P}<0.05$ was considered to indicate a statistically significant difference.

\section{Results}

Preliminary imaging results and clinical data of patients. According to the clinical data and preliminary imaging data of patients, incidence rate of child patients with a family disease history was higher than that of patients without a
Table II. Preliminary imaging results and clinical data of patients $(\mathrm{N}=374)$.

\begin{tabular}{ll}
\hline Parameters & $\mathrm{n}(\%)$ \\
\hline
\end{tabular}

\section{Sex}

Male

Female

Clinical manifestation

Painless abdominal mass

Abdominal mass and abdominal pain

Chest tightness and cough

Frequent urination and urinary urgency

Bone joint pain

Irregular fever

Enlargement of superficial lymph nodes

Tumor origin

Adrenal medulla

Retroperitoneum

Sympathetic chain in pelvic cavity

Posterior mediastinum

Inferior neck

Tumor lymph nodes metastasis (TNM)

Stage I

Stage II

Stage III

Place of residence

Urban area

$206(55.1)$

Rural area

168 (44.9)

Delivery mode

Eutocia

$172(46.0)$

Cesarean

$202(54.0)$

Family medical history

Yes

No

79 (21.1)

family disease history. No significant differences were found in sex, residence and delivery mode. The clinical symptoms were mainly irregular fever $(87.4 \%)$, and abdominal mass and abdominal pain (79.1\%). NB mainly originated from retroperitoneum (59.1\%) and adrenal medulla (34.0\%). The number of patients in the TNM stage III was the largest (49.2\%) (Table II).

Basic situations of tumor lesions. A total of 214 cases (57.2\%) showed tumor lesions with blurred edge, and 160 cases $(42.8 \%)$ 
Table III. Basic situations of tumor lesions $(\mathrm{N}=374)$.

\begin{tabular}{lr}
\hline Parameters & $\mathrm{n}(\%)$ \\
\hline Edge & \\
Blurred & $214(57.2)$ \\
Clear & $160(42.8)$ \\
Size & \\
$<6.0 \times 7.0 \times 7.5 \mathrm{~cm}$ & $178(47.6)$ \\
$\geq 6.0 x 7.0 \times 7.5 \mathrm{~cm}$ & $196(52.4)$ \\
Form & $71(19.0)$ \\
Oval or quasi-circular shape & $303(81.0)$ \\
Irregular shape & \\
\hline & \\
\hline Table IV. Tumor calcification $(\mathrm{N}=351)$. & $\mathrm{n}(\%)$ \\
\hline Calcification forms & $196(55.8)$ \\
\hline Granular & $67(19.1)$ \\
Mass & $52(14.8)$ \\
Flocky & $36(10.3)$ \\
\hline Patchy & \\
\hline
\end{tabular}

showed clear edge. Size of lesion ranged from $2.0 \times 4.0 \times 4.0 \mathrm{~cm}$ to $12.0 \times 14.0 \times 15.0 \mathrm{~cm}$. There were 178 cases $(47.6 \%)$ with the lesion size $>6.0 \times 7.0 \times 7.5 \mathrm{~cm}$ (median value), and 196 cases $(52.4 \%)$ with the lesion size $<6.0 \times 7.0 \times 7.5 \mathrm{~cm}$. Lesions with irregular shape were found in 303 cases $(81.0 \%)$, and lesions with oval or quasi-circular shape were found in 71 cases (19.0\%) (Table III).

Tumor density. CT plain scan showed the non-uniform density of tumor in 296 cases, and cystic changes and necrosis in lesions in 219 cases. Tumor calcification occurred in 351 cases, and granular calcification was found in 196 cases (55.8\%), and mass, flocky and patchy calcifications were found in other cases (Table IV). Enhanced scanning showed the non-uniform mild and moderate enhancement, and no enhancement was seen in regions with cystic changes and necrosis in lesions. Ninety four cases showed uniform low-density changes in lesions, 26 cases showed non-uniform mild and moderate enhancement, and 53 cases showed uniform mild enhancement.

Space-occupying effect and outward invasion of tumor. The space-occupying effect of retroperitoneal mass was the most obvious, in which the kidney was pressed to move, and its anteroposterior diameter became smaller. In 374 patients, there were 106 cases of axial rotation of kidney, 53 cases of enlargement of renal calyce and renal pelvis, 66 cases of elevation of liver position, 71 cases of pancreas translocation, 26 cases of gastrointestinal tract translocation, 17 cases of vascular translocation and 12 cases of bladder translocation, while 23 cases showed no significantly abnormal changes. Moreover, 211 cases had retroperitoneal lymphatic metastasis with soft tissue swelling in phrenic angle, abdominal aorta
Table V. Marker detection.

\begin{tabular}{lcrc}
\hline Marker & Positive (n) & Negative (n) & Positive rate $(\%)$ \\
\hline NES & 302 & 72 & 80.7 \\
CgA & 312 & 62 & 83.4 \\
S-100 & 171 & 203 & 45.7 \\
Syn & 374 & 0 & $100 \%$ \\
\hline
\end{tabular}

Table VI. Logistic regression analysis of the correlation between CT signs and pathological features.

\begin{tabular}{lccc}
\hline Features & P & OR & $95 \%$ CI \\
\hline Growth characteristics & 0.022 & 1.59 & $1.07-2.37$ \\
Calcification & 0.061 & 1.47 & $0.98-2.20$ \\
Degree of differentiation & 0.017 & 1.64 & $1.09-2.47$ \\
\hline
\end{tabular}

CT, computed tomography; OR, odds ratio; CI, confidence interval.

and renal hilum in image, and non-uniform annular enhancement or uniform enhancement in enhanced scanning. NB in right adrenal gland invaded the liver in 53 cases, invaded kidney in 26 cases, invaded psoas in 40 cases and invaded blood vessels in 32 cases, and the remaining cases showed no invasion. Sixty eight cases were accompanied by pleural thickening, 34 cases by pleural effusion, 36 cases by tracheal compression, 38 cases by rib compression, and 40 cases by tumor invading into vertebral canal. Bone metastasis occurred in 182 cases, liver metastases in 28 cases and brain metastases in 35 cases.

Pathological section examination. Pathological examinations for 374 child patients showed that the tumor cells were mostly oval or quasi-circular in shape. Nuclei were deeply stained. The amount of cytoplasm was reduced, and cytoplasm was arranged like the petals of chrysanthemum. Bleeding, necrosis and calcium deposition were also observed. Marker Syn showed the highest positive rate $(100 \%)$, followed by CgA (83.4\%) (Table V).

Correlation between CT signs and pathological features. Logistic regression analysis showed that tumor growth characteristics were closely correlated with the degree of differentiation and pathological changes $(p<0.05)$, while no significant correlations with calcification were observed ( $\mathrm{p}>0.05)$, (Table VI).

\section{Discussion}

$\mathrm{NB}$, as a common type of malignant tumor, is mainly composed of neuroblasts. NB mainly originates from any neural crest in the sympathetic nervous system (9). In the clinical treatment of NB, targeted operation is often performed based on its risk factors, and the low-risk group only needs tumor resection, 
while the moderate-risk group requires peripheral organ repair after the removal of tumor tissues, and the high-risk group should receive stem cell transplantation; high-dose chemotherapy, and general radiotherapy after primary tumor tissues, and metastatic lesions are removed as much as possible (10). Due to the lack of typical symptoms in the early stage, NB can be easily ignored, and the optimal treatment timing may be missed. Besides, prognosis is different in patients with different types of NB, due to the heterogeneity of this disease (11). The treatment process of NB is complicated, and the postoperative survival rate is low, causing physical and mental harm and economic loss to patients and their families. In view of this, correlations between CT signs and pathological features of patients with NB were analyzed in this study, with an expectation of improving the early diagnosis and treatment of NB.

According to the case analysis, child patients with a family disease history had a higher incidence rate, suggesting that the family medical disease may be a risk factor of NB. Sex, residence and delivery mode showed no significant effects on the occurrence of NB. Almeida et al (12) reported that some genetic predisposing factors may be related to the pathogenesis of NB, and somatic mutations of lymphoma hormone may be one of the pathogeneses of familial NB. However, no previous study clarifies the pathogenesis of NB, so further study is needed. Clinical symptoms of NB mainly include irregular fever, abdominal distension and abdominal pain, which are related to the tumor site in patients. Retroperitoneal NB is the most common type, so the clinical manifestation is mostly abdominal mass (13). Krytska et al (14) showed that most parents of NB patients do not have sufficient medical knowledge, and the disease is usually ignored or misdiagnosed as abdominal distension and fever. Patients are often in TMN stage III, suggesting that parents should be educated with the clinical knowledge of early symptoms of NB to improve early diagnosis, and increase survival rate. Plain CT scan usually shows an irregular large soft tissue mass, unclear edge, no obvious capsule, more cells with less mesenchyme, high degree of malignancy, rapid development, proneness to cystic changes and necrosis in imaging of NB patients (15). In this study, 351 out of 374 cases showed calcification mainly with granular shape, but flocky and patchy calcification was also observed. The mass is large, and often extends across the midline to the opposite side to wrap the retroperitoneal large vessels. Metastasis occurs frequently in early stage of NB (16). In this study, there were 245 out of 374 cases of metastasis, among which there were 211 cases of retroperitoneal lymphatic metastasis. Therefore, the unexplained fever, abdominal distension, abdominal pain, chest tightness and cough in children may indicate existence of NB, and they should be treated as soon as possible once diagnosed. Besides, 174 showed invasion to the vertebral canal or liver. Enhancement scan showed non-uniform mild or moderate enhancement, but there was no enhancement in regions with cystic changes and necrotic area inside the lesions, which can be used as a criterion to determine the degree of tumor wrapped vessels and the integrity of kidney (17). For patients without cystic changes and necrosis, imaging results mostly showed less tumor cells with more mesenchyme and lower degree of malignancy. CT scan showed low density and clearer border, as well as mild or no enhancement in enhanced scanning, indicating that CT features are closely correlated with the tumor cell components. NB is a kind of malignant tumor with aggressive growth along the paravertebral sympathetic chain, and NB usually invaded or wrapped around the surrounding tissues or organs with spindle- or irregular-shapes (18). During development, NB can easily cross the midline, and invade the spinal canal along the neural tube, thus expanding the intervertebral foramen, and causing damage to the vertebral body (19). In this investigation 151 out of 374 patients with NB showed invasive growth, and CT showed the invasion to surrounding organs or tissues, and wrapped surrounding blood vessels was the main signs. There were 114 cases of NB showing limited mass compression and a low degree of malignancy in surrounding tissues, indicating that the tumor growth characteristics are closely correlated with the tumor differentiation degree. Grey white soft substances in tissue sections of patients with NB could be seen by the naked eye, and the microscopy showed round or quasi-circular shape with diffuse distribution or densely-arranged mass and unclear cell boundary. Moreover, there was less cytoplasm, uniform distribution of chromatin, petal-like arrangement, large and deeply-stained nuclei, prominent nucleoli, nuclear fission and fibrous trabecula in tumor mesenchyme. The positive rates of NES, CgA, S-100 and Syn in NB patients were $80.7 \%, 83.4 \%, 45.7 \%$ and $100 \%$, respectively. NES, $\mathrm{CgA}, \mathrm{S}-100$ and Syn expression are observed in NB (20), and NES, CgA and Syn are neuroendocrine tumor markers with high specificity, so they can be used as important indicators to judge the severity of NB. The positive rates of markers were also basically consistent with the pathological stage and malignant degree of NB, indicating the promising application values of above markers in judging the severity of NB.

In conclusion, patients with distal mediastinal tumor identified via $\mathrm{CT}$ examination, accompanied by calcification, and invasion to surrounding tissues may suffer from $\mathrm{NB}$, and the characteristics of tumor growth are closely correlated with tumor differentiation degree.

\section{Acknowledgements}

Not applicable.

Funding

No funding was received.

\section{Availability of data and materials}

The datasets used and/or analyzed during the present study are available from the corresponding author on reasonable request.

\section{Authors' contributions}

$\mathrm{XZ}, \mathrm{CL}$ and $\mathrm{CX}$ were devoted to collecting and interpreting the data. XZ drafted this manuscript. $\mathrm{XH}$ and $\mathrm{XY}$ revised it critically for important intellectual content. XH, XY and QL were responsible for the conception and design of the study. All authors read and approved the final manuscript. 


\section{Ethics approval and consent to participate}

This study was approved by the Ethics Committee of Xuzhou Children's Hospital (Xuzhou, Jiangsu). Signed informed consents were obtained from the guardians of the patients.

\section{Patient consent for publication}

Not applicable.

\section{Competing interests}

The authors declare that they have no competing interests.

\section{References}

1. Irwin MS and Park JR: Neuroblastoma: Paradigm for precision medicine. Pediatr Clin North Am 62: 225-256, 2015.

2. Pinto NR, Applebaum MA, Volchenboum SL, Matthay KK, London WB, Ambros PF, Nakagawara A, Berthold F, Schleiermacher G, Park JR, et al: Advances in risk classification and treatment strategies for neuroblastoma. J Clin Oncol 33: 3008-3017, 2015.

3. Peifer M, Hertwig F, Roels F, Dreidax D, Gartlgruber M, Menon R, Krämer A, Roncaioli JL, Sand F, Heuckmann JM, et al: Telomerase activation by genomic rearrangements in high-risk neuroblastoma. Nature 526: 700-704, 2015.

4. Franscini LC, Vazquez-Montes M, Buclin T, Perera R, Dunand M, Grouzmann E and Beck-Popovic M: Pediatric reference intervals for plasma free and total metanephrines established with a parametric approach: Relevance to the diagnosis of neuroblastoma Pediatr Blood Cancer 62: 587-593, 2015.

5. Shinagawa T, Kitamura T, Katanoda K, Matsuda T, Ito Y and Sobue T: The incidence and mortality rates of neuroblastoma cases before and after the cessation of the mass screening program in Japan: A descriptive study. Int J Cancer 140: 618-625, 2016.

6. Armstrong GT, Chen Y, Yasui Y, Leisenring W, Gibson TM, Mertens AC, Stovall M, Oeffinger KC, Bhatia S, Krull KR, et al: Reduction in late mortality among 5-Year survivors of childhood cancer. N Engl J Med 374: 833-842, 2016.

7. Huang SY, Bolch WE, Lee C, Van Brocklin HF, Pampaloni MH, Hawkins RA, Sznewajs A, DuBois SG, Matthay KK and Seo Y: Patient-specific dosimetry using pretherapy $\left.{ }^{124} \mathrm{I}\right] \mathrm{m}$-iodobenzylguanidine $\left(\left[{ }^{124} \mathrm{I}\right] \mathrm{mIBG}\right)$ dynamic PET/CT imaging before $\left[{ }^{131} \mathrm{I}\right]$ mIBG targeted radionuclide therapy for neuroblastoma. Mol Imaging Biol 17: 284-294, 2015.

8. Kong G, Hofman MS, Murray WK, Wilson S, Wood P, Downie P, Super L, Hogg A, Eu P and Hicks RJ: Initial experience with gallium-68 DOTA-octreotate PET/CT and peptide receptor radionuclide therapy for pediatric patients with refractory metastatic neuroblastoma. J Pediatr Hematol Oncol 38: 87-96, 2016.
9. Louis CU and Shohet JM: Neuroblastoma: Molecular pathogenesis and therapy. Annu Rev Med 66: 49-63, 2015.

10. Kushner BH, Ostrovnaya I, Cheung IY, Kuk D, Modak S, Kramer K, Roberts SS, Basu EM, Yataghene K and Cheung N-KV: Lack of survival advantage with autologous stem-cell transplantation in high-risk neuroblastoma consolidated by anti-GD2 immunotherapy and isotretinoin. Oncotarget 7: 4155-4166, 2016.

11. de Oliveira MR, Schuck PF and Bosco SMD: Tanshinone I induces mitochondrial protection through an Nrf2-dependent mechanism in paraquat-treated human neuroblastoma SH-SY5Y cells. Mol Neurobiol 54: 4597-4608, 2017.

12. Almeida VR, Vieira IA, Buendia M, Brunetto AT, Gregianin LJ, Brunetto AL, Klamt F, de Farias CB, Abujamra AL, Lopez PLDC, et al: Combined treatments with a retinoid receptor agonist and epigenetic modulators in human neuroblastoma cells. Mol Neurobiol 54: 7610-7619, 2017.

13. Radogna F, Cerella C, Gaigneaux A, Christov C, Dicato M and Diederich M: Cell type-dependent ROS and mitophagy response leads to apoptosis or necroptosis in neuroblastoma. Oncogene 35: 3839-3853, 2016

14. Krytska K, Ryles HT, Sano R, Raman P, Infarinato NR, Hansel TD, Makena MR, Song MM, Reynolds CP and Mossé YP: Crizotinib synergizes with chemotherapy in preclinical models of neuroblastoma. Clin Cancer Res 22: 948-960, 2016.

15. Esposito MR, Aveic S, Seydel A and Tonini GP: Neuroblastoma treatment in the post-genomic era. J Biomed Sci 24: 14, 2017.

16. Young K, Alusi G and Korbonits M: Novel targeted treatment combinations for malignant neuroendocrine tumour olfactory neuroblastoma. Endoc Abstr 38: 153, 2015.

17. Lattanzio F, Carboni L, Carretta D, Candeletti S and Romualdi P: Treatment with the neurotoxic A $\beta$ (25-35) peptide modulates the expression of neuroprotective factors Pin1, Sirtuin 1, and brainderived neurotrophic factor in SH-SY5Y human neuroblastoma cells. Exp Toxicol Pathol 68: 271-276, 2016.

18. George SL, Falzone N, Chittenden S, Kirk SJ, Lancaster D, Vaidya SJ, Mandeville H, Saran F, Pearson AD, Du Y, et al: Individualized ${ }^{131} \mathrm{I}-\mathrm{mIBG}$ therapy in the management of refractory and relapsed neuroblastoma. Nucl Med Commun 37: 466-472, 2016

19. Bagatell R and Cohn SL: Genetic discoveries and treatment advances in neuroblastoma. Curr Opin Pediatr 28: 19-25, 2016.

20. Soriano A, París-Coderch L, Jubierre L, Martínez A, Zhou X, Piskareva O, Bray I, Vidal I, Almazán-Moga A, Molist C, et al: MicroRNA-497 impairs the growth of chemoresistant neuroblastoma cells by targeting cell cycle, survival and vascular permeability genes. Oncotarget 7: 9271-9287, 2016.

This work is licensed under a Creative Commons Attribution-NonCommercial-NoDerivatives 4.0 International (CC BY-NC-ND 4.0) License. 